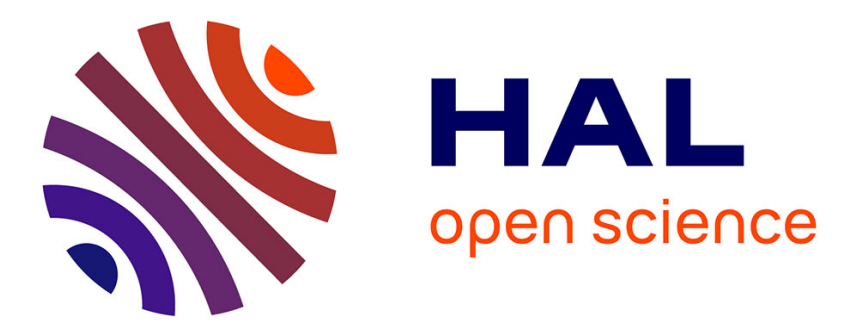

\title{
Bandgap inhomogeneity of MoS_2 monolayer on epitaxial graphene bilayer in van der Waals p-n junction
}

Zeineb Ben Aziza, Hugo Henck, Daniela Di Felice, Debora Pierucci, Julien

Chaste, Carl H. Naylor, Adrian Balan, Yannick J. Dappe, A.T. Charlie Johnson, Abdelkarim Ouerghi

\section{To cite this version:}

Zeineb Ben Aziza, Hugo Henck, Daniela Di Felice, Debora Pierucci, Julien Chaste, et al.. Bandgap inhomogeneity of MoS_2 monolayer on epitaxial graphene bilayer in van der Waals p-n junction. Carbon, 2016, 110, pp.396 - 403. 10.1016/j.carbon.2016.09.041 . cea-01490885

\section{HAL Id: cea-01490885 https://hal-cea.archives-ouvertes.fr/cea-01490885}

Submitted on 16 Mar 2017

HAL is a multi-disciplinary open access archive for the deposit and dissemination of scientific research documents, whether they are published or not. The documents may come from teaching and research institutions in France or abroad, or from public or private research centers.
L'archive ouverte pluridisciplinaire HAL, est destinée au dépôt et à la diffusion de documents scientifiques de niveau recherche, publiés ou non, émanant des établissements d'enseignement et de recherche français ou étrangers, des laboratoires publics ou privés. 


\title{
Bandgap inhomogeneity of $\mathrm{MoS}_{2}$ monolayer on epitaxial graphene bilayer in van der Waals $p-n$ junction
}

\author{
Zeineb Ben Aziza ${ }^{a}$, Hugo Henck ${ }^{a}$, Daniela Di Felice ${ }^{b}$, Debora Pierucci ${ }^{a}$, Julien Chaste ${ }^{a}$, \\ Carl H. Naylor ${ }^{\text {c }}$, Adrian Balan ${ }^{\text {c}}$, Yannick J. Dappe ${ }^{\text {b }}$, A.T. Charlie Johnson ${ }^{\text {c, }}$, \\ Abdelkarim Ouerghi ${ }^{\text {a, * }}$ \\ a Centre de Nanosciences et de Nanotechnologies, CNRS, Univ. Paris-Sud, Université Paris-Saclay, C2N - Marcoussis, 91460 Marcoussis, France \\ b SPEC, CEA, CNRS, Université Paris Saclay, CEA Saclay, 91191, Gif-sur-Yvette Cedex, France \\ c Department of Physics and Astronomy, University of Pennsylvania, 209S 33rd Street, Philadelphia, PA, 19104 6396, USA
}

\section{A R T I C L E I N F O}

\section{Article history:}

Received 6 June 2016

Received in revised form

13 September 2016

Accepted 17 September 2016

Available online 18 September 2016

\begin{abstract}
A B S T R A C T
Atomically thin $\mathrm{MoS}_{2}$ /graphene vertical heterostructures are promising candidates for nanoelectronic and optoelectronic technologies. In this work, we studied the optical and electronic properties of $n$ doped single layer $\mathrm{MoS}_{2}$ on p doped bilayer graphene vdW heterostructures. We demonstrate a non-uniform strain between two different orientation angles of $\mathrm{MoS}_{2}$ monolayer on top of epitaxial bilayer graphene. A significant downshift of the $E_{2 g}^{1}$ mode, a slight downshift of the $A_{1 g}$ mode, and photoluminescence shift and quenching are observed between two $\mathrm{MoS}_{2}$ monolayers differently oriented with respect to graphene; This could be mostly attributed to the strain-induced transition from direct to indirect bandgap in monolayer $\mathrm{MoS}_{2}$. Moreover, our theoretical calculations about differently-strained $\mathrm{MoS}_{2}$ monolayers are in a perfect accordance with the experimentally observed behavior of differently-oriented $\mathrm{MoS}_{2}$ flakes on epitaxial bilayer graphene. Hence, our results show that straininduced bandgap engineering of single layered $\mathrm{MoS}_{2}$ is dependent on the orientation angle between stacked layers. These findings could be an interesting novel way to take advantage of the possibilities of $\mathrm{MoS}_{2}$ and deeply exploit the capabilities of $\mathrm{MoS}_{2}$ /graphene van der Waals heterostructures.
\end{abstract}

() 2016 Elsevier Ltd. All rights reserved.
Ever since the technique to isolate stable single-layer graphene was reported, the study of two-dimensional (2D) materials has gained a lot of research interest. Hence, a broad family of 2D materials like graphene, transition metal dichalcogenides (TMDCs), and topological insulators have been explored so far [1]. For the sake of exploring their fundamental interfacial interactions and conceiving novel electronic functionalities, vertical heterostructures composed of 2D materials stacked together by van der Waals (vdW) forces have attracted a great attention lately [2]. Among these combined structures, the $\mathrm{MoS}_{2}$ /graphene heterostructure, among other heterostructures combining graphene with different layered 2D materials [3], shows a promising potential for next generation nanoelectronic and optoelectronic devices thanks to the outstanding carrier mobilities on one hand and the excellent optical responsivities on the other hand [4-6].

\footnotetext{
* Corresponding author.

E-mail address: abdelkarim.ouerghi@lpn.cnrs.fr (A. Ouerghi).
}

However, research work about the structural quality, the band alignment and the optical emission properties of $\mathrm{MoS}_{2}$ /graphene or graphene/MoS 2 vdW heterostructures are still limited. The exploration of such characteristic properties are fundamental to make possible the assembling of $\mathrm{MoS}_{2}$ with graphene, pave the way for the realization of a well-ordered $\mathrm{MoS}_{2}$ /graphene structure, and enable opportunities for a wide spectrum of optoelectronic functionalities. Inspirations of this present work arise from the fact that some of the theoretical calculations have predicted the crossover between a direct and indirect bandgap of $\mathrm{MoS}_{2}$ originating from the modification of interlayer orientation [7-9]. Since the properties of $\mathrm{MoS}_{2}$ /graphene heterostructure depend strongly on the quality of the interface between the underlying substrate and the top-layer as well as the interlayer orientation, the development of such van der Waals (vdW) heterostructure should start with a high quality substrate material such as graphene layer. Among various graphene substrates, epitaxial graphene (EG) on silicon carbide ( $\mathrm{SiC}$ ) provides several potential advantages for designing such heterostructures including high electronic mobility, tunable substrate coupling, 
wafer-scale process ability, and highly ordered crystalline structure that can template commensurate growth.

Here, we demonstrate that the orientation of $\mathrm{MoS}_{2}$ with respect to graphene, used as an underlying substrate, can affect the properties of $\mathrm{MoS}_{2}$ monolayer likely due to the variation of the strain level sustained by the $\mathrm{MoS}_{2}$ flakes. Raman spectroscopy and photoluminescence measurements were performed in order to gain a better insight into the changes observed in $\mathrm{MoS}_{2}$ optical properties. A significant downshift of the $\mathrm{E}^{1}{ }_{2 \mathrm{~g}}$ mode, and a slight downshift of the $\mathrm{A}_{1 \mathrm{~g}}$ mode are observed for $\mathrm{MoS}_{2}$ flake with different orientations, suggesting different strain levels supported by the different flakes. Moreover, a PL quenching occurred for the flake undergoing higher tensile strain which may bring about the transition from direct to indirect bandgap in monolayer $\mathrm{MoS}_{2}$. These results show that $\mathrm{MoS}_{2}$ bandgap structure is strongly dependent on the $\mathrm{MoS}_{2}$ orientation with respect to graphene. To our knowledge, the dependency of $\mathrm{MoS}_{2}$ band structure with the orientation of $\mathrm{MoS}_{2}$ on $\mathrm{p}$ doped epitaxial graphene has not been previously demonstrated experimentally. These findings could open the door for alternative techniques to tune $\mathrm{MoS}_{2}$ electronic properties for new nanoelectronic applications.

\section{Results and discussion}

We used highly crystalline $\mathrm{MoS}_{2}$ flakes grown by chemical vapor deposition (CVD) [10]. This CVD approach was adopted since it allows obtaining large $\mathrm{MoS}_{2}$ flakes with high electrical and optical properties. A PMMA assisted method was employed to transfer the CVD grown $\mathrm{MoS}_{2}$ on epitaxial monolayer graphene on SiC. Typical single-crystal domains with an equilateral triangle shape were obtained by the CVD growth procedure of $\mathrm{MoS}_{2}$ on $\mathrm{SiO}_{2}$. The $\mathrm{MoS}_{2}$ domains transferred onto the graphene retain their triangular shapes with lateral sizes of $\sim 20$ to $\sim 200 \mu \mathrm{m}$. To further clean the surface and interface of the $\mathrm{MoS}_{2}$ /graphene heterostructure, we annealed the samples at $\mathrm{T}=350{ }^{\circ} \mathrm{C}$ for $60 \mathrm{~min}$ in UHV (base pressure below $\mathrm{P} \sim 10^{-10}$ mbar).

The graphene used in this work was obtained by an annealing of a $4 \mathrm{H}-\mathrm{SiC}(0001)$ substrate followed by a hydrogenation process, as described in materials and method section. With this process we obtained a quasi-freestanding $p$ doped bilayer graphene [11-13]. This quasi freestanding graphene presents a uniform thickness over a large area and a lower corrugation with respect to epitaxially grown graphene, representing a better template for 2D materials.

By making use of several characterization techniques, we try to find out the effect of the relative position of $\mathrm{MoS}_{2}$ monolayers, with respect to graphene, on the interlayer interactions between the two-stacked materials or in other words study the impact of changing the orientation on the properties of $\mathrm{MoS}_{2}$. The quality of this heterostructure on large scale was first studied using HR-XPS, STEM and micro-Raman spectroscopy. The Raman spectra comparing the pristine graphene (black curve) and the $\mathrm{MoS}_{2}$ /graphene heterostructure (blue curve) on a wavenumber range between 300 and $2800 \mathrm{~cm}^{-1}$ are shown in Fig. 1(a). The graphene layer has several overtone peaks in the $1000 \mathrm{~cm}^{-1}$ to $2000 \mathrm{~cm}^{-1}$ regime. The peak at $1522 \mathrm{~cm}^{-1}$ is considered to be an overtone of the L-point optical phonon from the SiC. The Raman signals from the graphene show prominent characteristic peaks at $1590 \mathrm{~cm}^{-1}$ (G) and $2720 \mathrm{~cm}^{-1}$ (2D), which give evidence of carbon $s p^{2}$ reorganization. The $G$ peak value of $1590 \mathrm{~cm}^{-1}$ could indicate strong $\mathrm{p}$ doping. The low intensity of the $\mathrm{D}$ peak (in comparison to the $\mathrm{G}$ peak), in both spectra, is a clear indication of zone-boundary phonons scattering on defects, allowed in ideal graphene due to the large wave-vector of the K-point phonon involved. Its absence is an indication of high quality of the $\mathrm{MoS}_{2}$ /graphene heterostructure. We can also see the broad 2D peak showing the bilayer structure of graphene. For $\mathrm{MoS}_{2}$ /graphene based heterostructure (black curve), two characteristic features arise within the wavenumber range between 370 and $420 \mathrm{~cm}^{-1}: \mathrm{E}_{2 \mathrm{~g}}{ }$ and $\mathrm{A}_{1 \mathrm{~g}}[14,15]$. The peak frequency difference between these $\mathrm{E}^{1}{ }_{2 \mathrm{~g}}$ and $\mathrm{A}_{1 \mathrm{~g}}$ vibrational modes is around $\sim 19-20 \mathrm{~cm}^{-1}$, which is in agreement with what was previously reported for $\mathrm{MoS}_{2}$ monolayer [16,17].

HR-XPS measurements were carried out for the pristine graphene and $\mathrm{MoS}_{2}$ /graphene heterostructure, as shown in Fig. 1(b). These XPS measurements performed on a wide energy range show the presence of the Mo 3p, Mo 3d and $S 2 p$ peaks (blue curve) beside the standard components present on epitaxial graphene on $\mathrm{SiC}, \mathrm{C}$ $1 s$, Si $2 s$, Si 2p, (black curve), signature of the $\mathrm{MoS}_{2}$ flakes on the graphene underlayer. No other element is detected in our spectra. The low intensity of O $1 s$ signal in the XPS spectra validates that the oxygen contamination has been removed by the annealing at $400{ }^{\circ} \mathrm{C}$, performed before all measurements, under UHV conditions. High resolution spectra for $\mathrm{C}$, Mo and $\mathrm{S}$ are also recorded in surface sensitive condition ( $\mathrm{h} v=340 \mathrm{eV}$ ) (Fig. 2(a)-(c)). The different components on the spectra were decomposed by a curve fitting procedure (see method). The experimental data points are displayed in dots and the red solid lines represent the envelope of the fitted components. The $\mathrm{C}$ 1s spectrum for a p-doped quasi free standing bilayer graphene is shown in Fig. 2(a). Only two components are present on the spectra [11] due to the bilayer graphene (G peak at binding energy $\mathrm{BE}=284.3 \mathrm{eV}$ ) and the $\mathrm{SiC}$ substrate $(\mathrm{BE}=282.6 \mathrm{eV})$. Respect to an as grown n-doped monolayer epitaxial graphene [18] the $G$ peak presents a shift of about $0.4 \mathrm{eV}$ to lower BE indicating a change in the doping, from $n$ to $p$, induced by the hydrogenation process [11]. Moreover, the SiC component is shifted of about $1 \mathrm{eV}$ to lower $\mathrm{BE}$, which confirms that the hydrogen bonds are present at the SiC surface inducing this band bending variation [10] confirming a complete decoupling of the buffer layer.

The Mo 3d peak presented in Fig. 2(b) and the $S$ 2p peak in Fig. 2(c) show the standard deconvolution expected for $\mathrm{Mo}^{4+}$ and a divalent sulfide ions $\left(\mathrm{S}^{2-}\right)$ in $\mathrm{MoS}_{2}$ [19]. The Mo 3d $\mathrm{d}_{5 / 2} \mathrm{BE}=229.7 \mathrm{eV}$ $\left(\right.$ Mo $3 \mathrm{~d}_{3 / 2}=232.8 \mathrm{eV}$ ) and S $2 \mathrm{p}_{3 / 2} \mathrm{BE}=162.5 \mathrm{eV}(\mathrm{S} \mathrm{2p} 1 / 2$ $\mathrm{BE}=163.7 \mathrm{eV}$ ) indicate an intrinsic n-type doping of the $\mathrm{MoS}_{2}[20]$. This $n$ type doping is due to the presence of sulphur vacancies, an electron donating defect [21], at the edge of the $\mathrm{MoS}_{2}$ flakes. The signature of this defect is present in the Mo $3 \mathrm{~d}$ peak as a small component at a lower $\mathrm{BE}(\sim-0.5 \mathrm{eV})$ with respect to the main Mo $3 d_{5 / 2}$ peak. In both spectra, no signature of other bonds are present [19,22-25] (i.e. oxygen or carbon), indicating the absence of any inter-diffusion of contamination between the $\mathrm{MoS}_{2}$ and graphene layers. In order to investigate the interface, cross-sectional STTEM experiments were performed on $\mathrm{MoS}_{2}$ /epitaxial graphene as shown in Fig. 2(d). This cross-sectional view was observed along the (11-20) SiC zone axis. HR-TEM images reveal the thickness of the $\mathrm{MoS}_{2}$ layers and the detailed crystalline structure of the $\mathrm{MoS}_{2}$ and graphene layer. As observed from the HR-TEM images, the $\mathrm{MoS}_{2}$ /graphene heterostructure is predominantly composed by monolayer $\mathrm{MoS}_{2}$ and bilayer graphene. The interlayer separation is about $0.34 \pm 0.02 \mathrm{~nm}$, the $\mathrm{MoS}_{2}$ layers are atomically flat and form a continuous film. This image shows that the annealing procedure of $\mathrm{MoS}_{2}$ /graphene leads to clean and sharp interface without any structural defects.

All previous experiments were performed on $\mathrm{MoS}_{2} /$ bilayer graphene to study the interface quality between $\mathrm{MoS}_{2}$ and graphene without discussing the effect of the twist of $\mathrm{MoS}_{2}$ on graphene layers since this effect cannot be clearly detected using these techniques. In order to probe the effects of the twist on the electronic and the vibrational properties of the $\mathrm{MoS}_{2}$ layer, we carried out micro-photoluminescence mapping on the two orientations between $\mathrm{MoS}_{2}$ and graphene layer, and we also performed microRaman spectroscopy on our samples. The optical image in 
Fig. 3(a) shows triangular flakes of $\mathrm{MoS}_{2}$, a small and a big one on the graphene-underlying layer. The epitaxial graphene completely covers the $\mathrm{SiC}$ substrate following its topography. From this image we can estimate the orientation of the two $\mathrm{MoS}_{2}$ flakes with respect to the graphene underlayer. In fact the $\Gamma \mathrm{M}$ direction of the first graphene Brillouin zone is oriented parallel to the step edge of the $\mathrm{SiC}$ substrate and the $\Gamma \mathrm{K}$ direction of the $\mathrm{MoS}_{2}$ Brillouin zone is parallel to the side of the flakes (more details are given in Fig. S1). Then we found a mismatch angle of $33^{\circ} \pm 2^{\circ}$ for the small flake and $4^{\circ} \pm 2^{\circ}$ for the big one. Moreover based on the contrast, one can tell that the $\mathrm{MoS}_{2}$ flakes are monolayers except for one lighter triangular spot (inside the big flake), which corresponds to multilayers of $\mathrm{MoS}_{2}$. A 3D schematic presentation is provided in Fig. 3(b) to better explain the considered $\mathrm{MoS}_{2} /$ graphene heterostructure. It should be noted that before performing the PL and Raman measurements, the samples were pumped in ultra-high vacuum for few days and then annealed at $300{ }^{\circ} \mathrm{C}$ for about $30 \mathrm{~min}$ to ensure the total removal of eventual impurities that may affect our results.

Fig. 3(c) and (d) illustrate the PL data carried on the studied sample; the uniform color within one single flake attest the absence of cracks and the high quality of our $\mathrm{MoS}_{2}$. From the PL map recorded from the single layer $\mathrm{MoS}_{2}$ regions in Fig. 3(c) one can notice the darker color of the PL intensity of the big flake or in other words, the PL intensity for the big flake is sharply decreased compared to the small flake. From the PL spectra in Fig. 3(d), we can see that the PL of the small flake is dominated by a strong peak observed at around $1.85 \mathrm{eV}$ reflecting the direct bandgap at the $\mathrm{K}$ point of the Brillouin zone. Another small peak appears at about $2 \mathrm{eV}$ in both PL spectra and corresponds to the valence band spinorbit coupling of $\mathrm{MoS}_{2}$ [26]. The ratio between the PL intensities of the two flakes is of about $3 / 2$; this behavior is similar to what we observed for monolayer and bilayer $\mathrm{MoS}_{2}[17,27,28]$.

These values of the bandgap, determined by PL spectroscopy

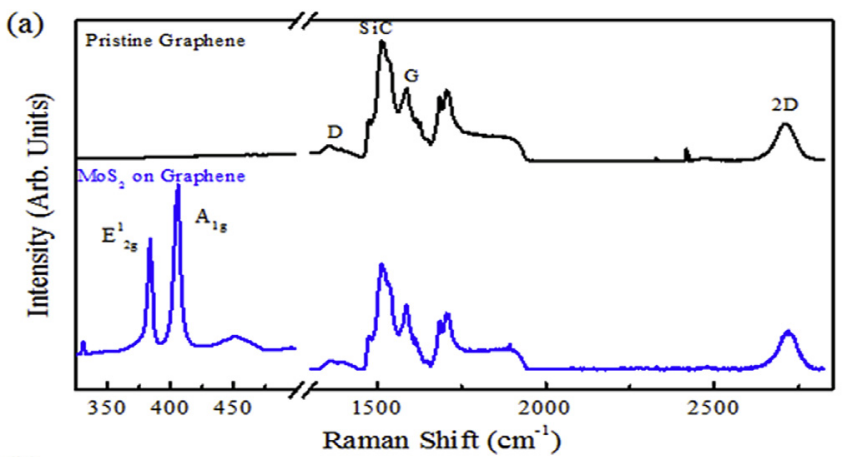

(b)

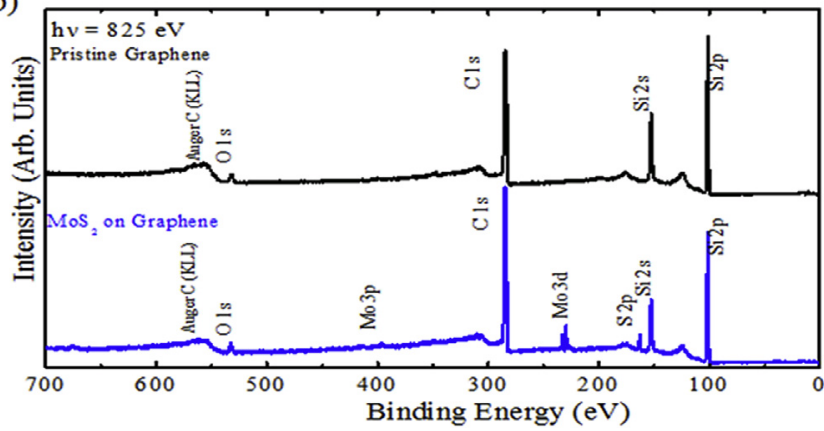

Fig. 1. (a) Large Raman spectra extending on a wavenumber range from 300 to $2800 \mathrm{~cm}^{-1}$ of pristine epitaxial graphene (black line) and $\mathrm{MoS}_{2}$ on epitaxial graphene (blue line), (b) HR- XPS spectrum on a wide energy range collected at a photon energy $\mathrm{h} v=825 \mathrm{eV}$ of pristine graphene (black curve) and of $\mathrm{MoS}_{2}$ on epitaxial graphene (blue curve). (A colour version of this figure can be viewed online.) and known as optical bandgap, are different from that determined by electronic transport due to the exciton binding energy [29]. As shown in the graph in Fig. 3(d), we get band gap values of $1.85 \mathrm{eV}$ and $1.79 \mathrm{eV}$ for the small and big flakes respectively; these values are similar to that reported for $\mathrm{MoS}_{2}$ monolayers. However, we notice as well a bandgap decrease of about $60 \mathrm{meV}$ in the case of the big flake yet both studied flakes represent single layers of $\mathrm{MoS}_{2}$ and they are characterized under the same conditions. Therefore, several hypotheses can be made to understand the origin of this change in $\mathrm{MoS}_{2}$ bandgap.

i) The presence of eventual residues at the interface between graphene and $\mathrm{MoS}_{2}$ and/or the impurities left after the resist assisted transfer process of $\mathrm{MoS}_{2}$ on graphene can probably induce doping in $\mathrm{MoS}_{2}$ and change its PL spectra [30]. The TEM image and the XPS data in Fig. 2 as well as the band structure of $\mathrm{MoS}_{2}$ /graphene provided in Figures S2 and S3(a) and (b) confirm that our samples are of high quality, free from cracks and contaminations since they are carefully pumped and annealed. Therefore, the change of the PL intensity cannot be associated to the presence of defective sites or contaminations [31,32].

ii) The quench of PL intensity and the energy shift can be attributed to the transition from negative trions to neutral excitons (at higher energy). This assumption cannot be considered true in our case: first, if such a transition exists, we would observe a shift to higher energy. Moreover, the observed shift is more important than the difference between $A$ peak and $\mathrm{A}^{-}$peak (typically this distance is about 20-50 meV) [33,34]. A sample of $\mathrm{MoS}_{2}$ on $\mathrm{SiO}_{2}$, whose SEM image is shown in Fig. S4, was prepared to discuss this point. The graphs, shown in Fig. S5, correspond to quasi freestanding $\mathrm{MoS}_{2}, \mathrm{MoS}_{2}$ transferred on $\mathrm{SiO}_{2}$ (used as a reference), and $\mathrm{MoS}_{2}$ transferred on graphene with different orientations. It is worth pointing out that the peak A corresponding to the excitons is notably observable only in quasifreestanding $\mathrm{MoS}_{2}$ [35] and that the splitting of peaks A and $\mathrm{A}^{-}$is not apparent when $\mathrm{MoS}_{2}$ is supported by a substrate. From these graphs, we clearly see that the shift is likely due a change in the $\mathrm{MoS}_{2}$ bandgap rather than a trion-exciton transition.

iii) The decrease of the PL intensity can be explained by a bandgap transition from a direct to indirect bandgap since it occurs with an energy shift in the PL peak [36] (this shift cannot be observed in the case where charge transfer occurs). The possible band structure change from direct to indirect band gap in monolayer $\mathrm{MoS}_{2}$ could be translated by the fact that the modification of the orientation angle of the different flakes with respect to graphene substrate may induce a variation of the properties of $\mathrm{MoS}_{2}$.

Hence, the latter explanation iii) seems to be suitable to justify our observations. To support this argument, we conducted microRaman experiments and density functional theory (DFT) calculations. Micro-Raman spectroscopy was employed here to study the changes of the vibrational modes of $\mathrm{MoS}_{2}$ flakes transferred on epitaxial graphene with different angles ( $4^{\circ}$ and $33^{\circ}$, respectively) and to investigate the strain levels supported by $\mathrm{MoS}_{2}$ flakes if any. As mentioned, there are two typical modes of $\mathrm{MoS}_{2}$ within the wavenumber range between 370 and $420 \mathrm{~cm}^{-1}: \mathrm{E}_{2 \mathrm{~g}}^{1}$, corresponding to the in-plane displacements and $\mathrm{A}_{1 \mathrm{~g}}$ corresponding to the outof-plane displacements of Mo and $\mathrm{S}$ atoms in the $\mathrm{MoS}_{2}$ films [37,38]. Fig. 4(a) and (b) show the Raman maps of the characteristic peak positions of the $\mathrm{E}_{2 \mathrm{~g}}^{1}$ and $\mathrm{A}_{1 \mathrm{~g}}$ modes of $\mathrm{MoS}_{2}$ measured on monolayer $\mathrm{MoS}_{2}$ flakes with different orientations on epitaxial 
(a)

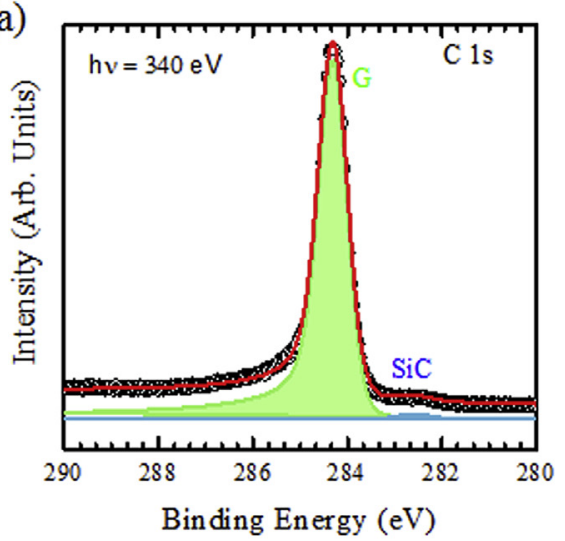

(c)

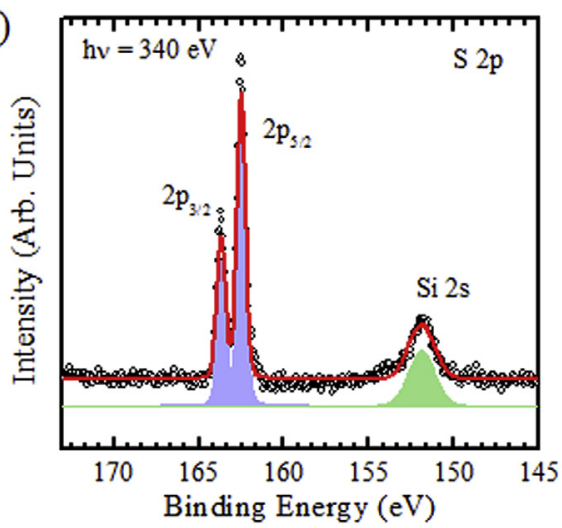

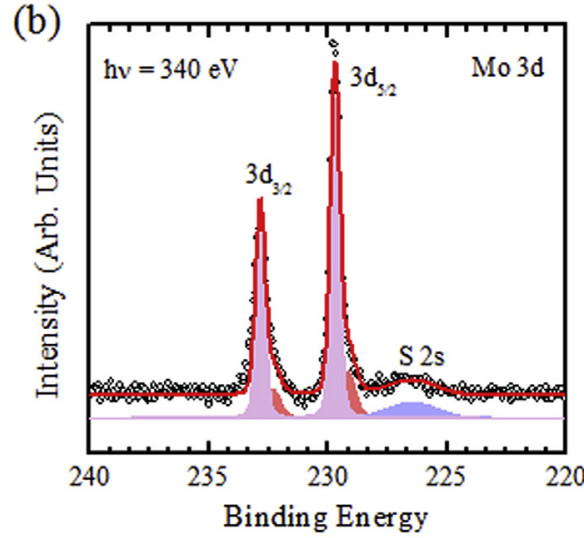

(d)

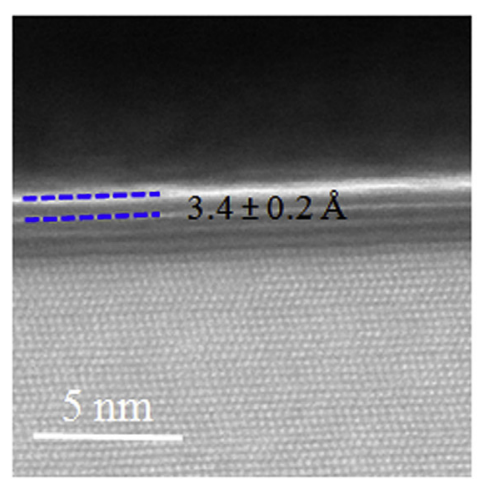

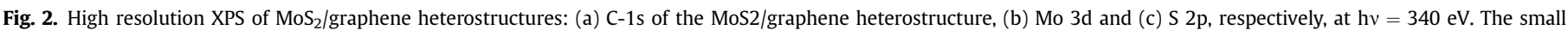

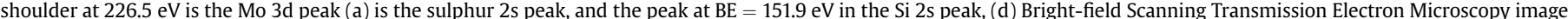
of $\mathrm{MoS}_{2}$ monolayer on epitaxial graphene. (A colour version of this figure can be viewed online.)

bilayer graphene. We clearly see that, for the big flake, the most prominent Raman peaks $E_{2 g}^{1}$ and $A_{1 g}$ have a brighter color indicating that both peaks shift to lower frequency $(\omega)$. From the Raman spectra in Fig. 4(c) measured, using a laser spot whose diameter is about $1 \mu \mathrm{m}$, in the centers of the small and big MoS 2 flakes (red and blue curves, respectively), we can obviously see that both peaks corresponding to the big flake are red-shifted. These downshifts can be explained based on the difference in the tensile strain sustained by $\mathrm{MoS}_{2}$ flakes $[37,39,40]$ rather than a difference in the doping levels caused by a dissimilarity in charge transfer for the two systems (if doping occurred, we would observe an up-shift of the $A_{1 g}$ mode while the $E^{1}{ }_{2 g}$ mode remains unchanged [29,41] since graphene is p-doped, but this not the case here). The atomic displacements of these two modes are illustrated in Fig. 4(d).

However, the shift in the $\mathrm{E}_{2 \mathrm{~g}}^{1}$ mode $\left(\sim 2.5 \mathrm{~cm}^{-1}\right)$ is more important than in the $\mathrm{A}_{1 \mathrm{~g}}$ mode $\left(\sim 1.2 \mathrm{~cm}^{-1}\right)$ attesting that the $\mathrm{A}_{1 \mathrm{~g}}$ mode shows weaker orientation dependence of $A_{1 g}$ mode than the $E_{2 g}^{1}$. This ratio between the shifts of $E_{2 g}^{1}$ and $A_{1 g}$ is in accordance with the results reported on the effect of uniform biaxial strain on the Raman spectrum of $\mathrm{MoS}_{2}$ [29]. Meanwhile, the widths of the peaks, calculated by means of a Lorentzian fitting, are barely unchanged. Based on the Raman shift values of the $E^{1}{ }_{2 g}$ mode, we can estimate the strain induced by varying the angle between the transferred $\mathrm{MoS}_{2}$ and graphene used as a supporting substrate using the following formula $[42,43]$ : $\partial \varepsilon_{\text {biaxial }}=-\frac{1}{2 \gamma} \frac{\partial \omega}{\omega_{0}}$ where $\gamma$ is the Gruneisen parameter and it is equal to 0.54 for $E^{1}{ }^{2}$ peak [44] and $\partial \omega$ is the shift calculated by the difference between the frequency positions of the small flake and the big one, respectively (the small flake is selected as the reference flake). We also assume that the strain sustained by the $\mathrm{MoS}_{2}$ flakes is a biaxial uniform strain based on the fact that the contrast within one single flake of $\mathrm{MoS}_{2}$ illustrated in the map in Fig. 4(a) is uniform for a given orientation angle. We found a strain variation value approximately equal to $\partial \varepsilon_{\text {biaxial }} \approx 0.6 \pm 0.1 \%>0$ between respectively the small flake and the big flake. This implies that the big flake undergoes a more important uniform tensile strain than the small flake; this can be justified by the different orientation of these flakes grown and transferred on top of the epitaxial graphene in exactly identical conditions. This value matches well the observed bandgap variation of about $60 \mathrm{meV}$ for a strain of $0.6 \%$ as well as the results that have been reported so far [45].

In order to confirm our experimental observations and see how a tensile strain affects the $\mathrm{MoS}_{2}$, we performed theoretical calculations to see the evolution of the bandstructure of $\mathrm{MoS}_{2}$ monolayer upon applying uniform tensile biaxial strain. The application of strain was ensured by modifying the lattice constant of $\mathrm{MoS}_{2}$. As shown in Fig. 5, the position of the maximum of the valence band of $\mathrm{MoS}_{2}$ can be tuned effectively by uniform tensile biaxial strain variation. Similar theoretical observations [46] were also reported about tuning the properties of $\mathrm{MoS}_{2}$, subjected to various levels of misorientation-induced lattice strain, through varying the angle between graphene and $\mathrm{MoS}_{2}$. This suggests that tuning the interlayer orientation could induce a strain change in $\mathrm{MoS}_{2}$ which may cause variations of $\mathrm{MoS}_{2}$ bandstructure [32,47]. Thus, it seems possible to tune the bandstructure around the $\mathrm{K}$ point by reducing the energy of the direct band gap via selectively choosing different orientations of the considered $\mathrm{MoS}_{2}$ flakes. Other theoretical calculations reported the evolution of $\mathrm{MoS}_{2}$ bandstructure by varying 
(a)

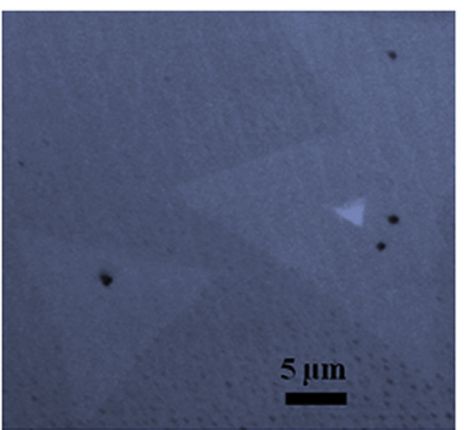

(c)

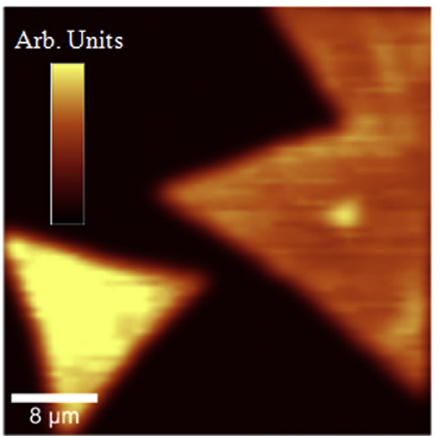

(b)

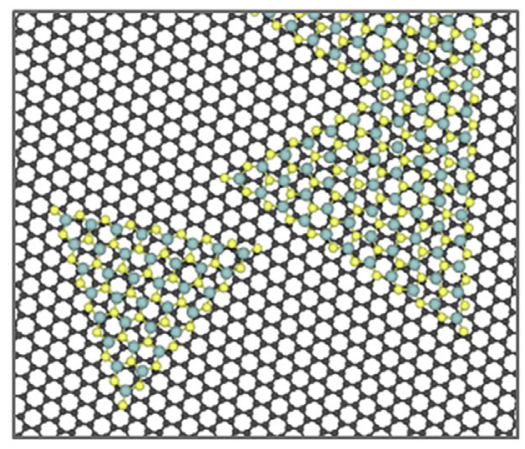

(d)

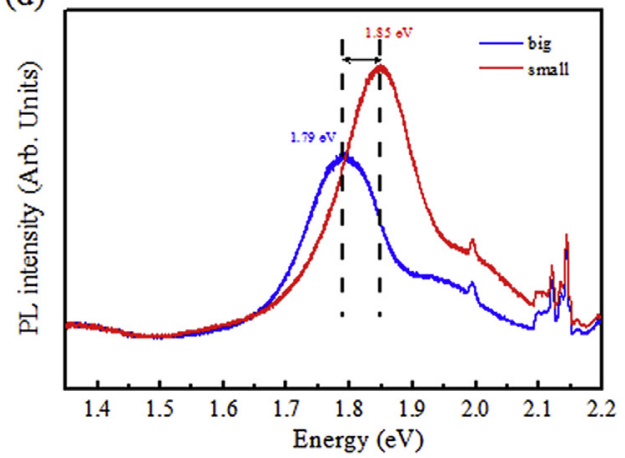

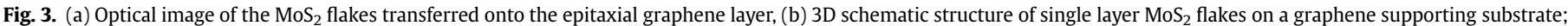

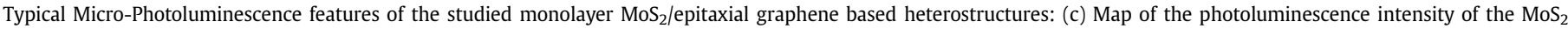

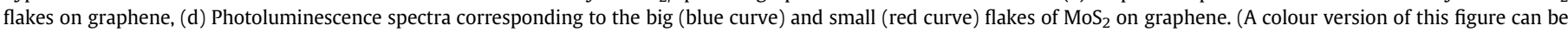
viewed online.)

the external electric field [48], a decrease of the tri-layer $\mathrm{MoS}_{2}$ gap was observed when increasing the electric field.

All these statements agree perfectly with the forgoing Raman and PL results and confirm the tunability of $\mathrm{MoS}_{2}$ monolayers bandgap upon changing the orientation angle of the single layered $\mathrm{MoS}_{2}$ with respect to epitaxial graphene bilayer. The reason why the different orientations of $\mathrm{MoS}_{2}$ flakes on top of epitaxial monolayer graphene can affect the $\mathrm{MoS}_{2}$ bandgap structure could be attributed to a change in the thickness of $\mathrm{MoS}_{2}$ as reported by A. Ebnonnasir et al. [7] who used DFT calculations to prove the impact of changing the orientation on the thickness of $\mathrm{MoS}_{2}$ on graphene when the two materials are brought together. They computed the bandgap of $\mathrm{MoS}_{2}$ for different values of its thickness and they found out that at equilibrium, the bandgap is direct, while a slight decrease in the thickness makes the bandgap smaller and indirect. This is also consistent with the findings of W. S. Yun et al. [49] who showed, using the first-principles calculations, that the tensile strain reduces the gap energy. Furthermore, W. Jin [8] used microprobe angle resolved photoemission spectroscopy to prove the dependency of $\mathrm{MoS}_{2}$ on the twist angle in graphene/MoS heterostructure. They affirm that the band structure of $\mathrm{MoS}_{2}$ becomes indirect except for $30^{\circ}$ twist angle with respect to graphene, meanwhile graphene properties remain intact.

According to all the reasons cited above, we can claim that the big flake is subjected to a more important tensile strain than the small flake, which causes a noticeable Raman frequency shift of the $E^{1}{ }_{2 g}$ mode and also renders the bandgap of the big flake smaller. It is also worth to mention that the origin of this strain may be generated upon cooling down of the sample; i.e. the lower temperature following the annealing of the $\mathrm{MoS}_{2}$ could induce an out of plane relaxation leading to a non-uniform strain distribution on the different flakes. Consequently, we found out that the relative orientation of $\mathrm{MoS}_{2}$ monolayer on top of graphene significantly influences the Raman spectra and the photoluminescence of $\mathrm{MoS}_{2}$ attesting a change in the value and the type of the bandgap of $\mathrm{MoS}_{2}$. This can be explained based on the fact that changing the orientation of $\mathrm{MoS}_{2}$ may bring about a change of the S-S interplanar distance likely because of the electron transfer away from the Mo-S bonds [7]. This could slightly affect the strain supported by $\mathrm{MoS}_{2}$ flakes as well as its thickness. These alterations would necessarily imply a noticeable change in the bandstructure of $\mathrm{MoS}_{2}$. Thus, by changing $\mathrm{MoS}_{2}$ monolayers orientation we observe a similar PL behavior as in the case of changing the number of layers of $\mathrm{MoS}_{2}$. Our theoretical calculations on the effect of tensile strain on the bandgap of $\mathrm{MoS}_{2}$ are in a good harmony with our experimental observations.

To conclude, we have reported that the orientation of $\mathrm{MoS}_{2}$ monolayer on bilayer graphene heterostructures affects the bandgap of $\mathrm{MoS}_{2}$. This suggests the dependency of the bandgap of $\mathrm{MoS}_{2}$ on the interfacial interactions within the heterostructures. Micro-Photoluminescence and micro-Raman spectroscopy have been shown to be of great promise for exploring the strain variation since the strain alters the crystalline symmetry and so the vibrational frequency of the typical modes of $\mathrm{MoS}_{2}$. Moreover, our findings represent a useful experimental support for the theoretical studies reported so far on the tunability of the bandstructure of $\mathrm{MoS}_{2}$ by varying the strain supported by $\mathrm{MoS}_{2}$ flakes. This implies that monitoring the twisting angles between vertically-stacked layers of $\mathrm{MoS}_{2}$ and graphene could be an efficient route for their electronic properties engineering. These interesting results can be useful to pave the way to reveal new approaches for strain engineering, to modulate the electronic properties of $\mathrm{MoS}_{2}$, and to design new layered structures with tunable optoelectronic functionalities. 
(a)

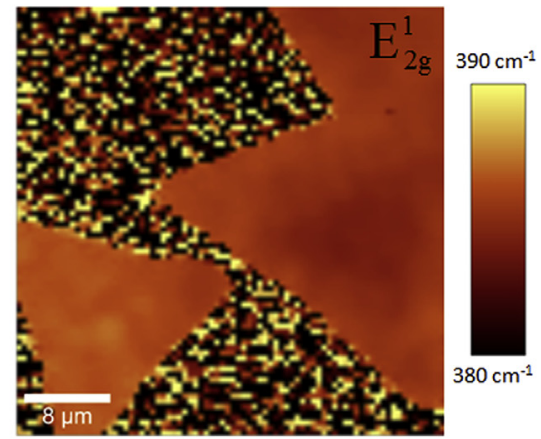

(c)

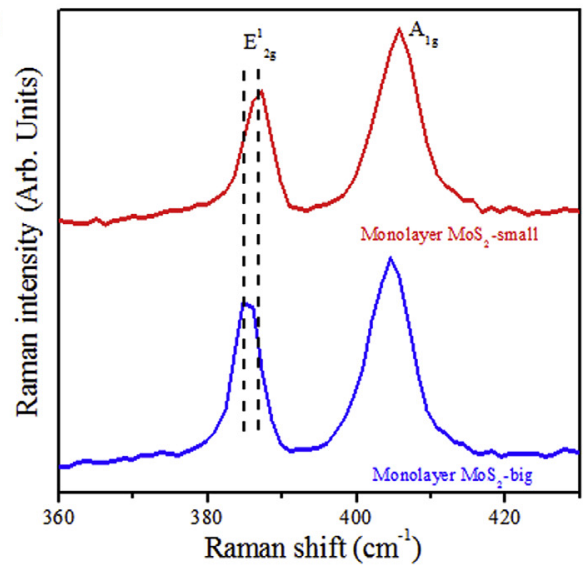

(b)

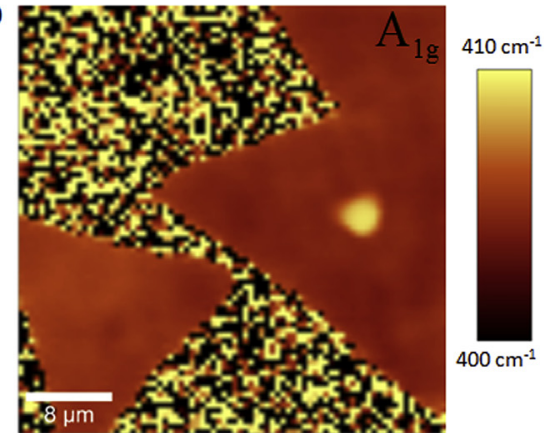

(d)

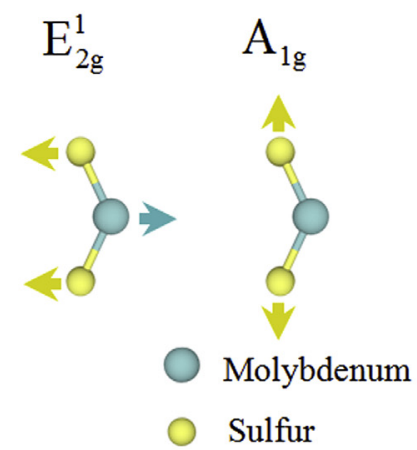

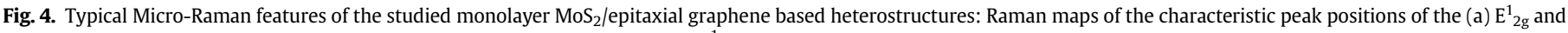

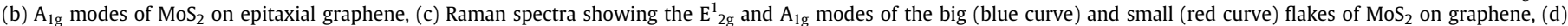
Schematic of the atomic displacements of the Raman modes $\mathrm{E}_{2 \mathrm{~g}}$ and $\mathrm{A}_{1 \mathrm{~g}}$ of $\mathrm{MoS}_{2}$. (A colour version of this figure can be viewed online.)

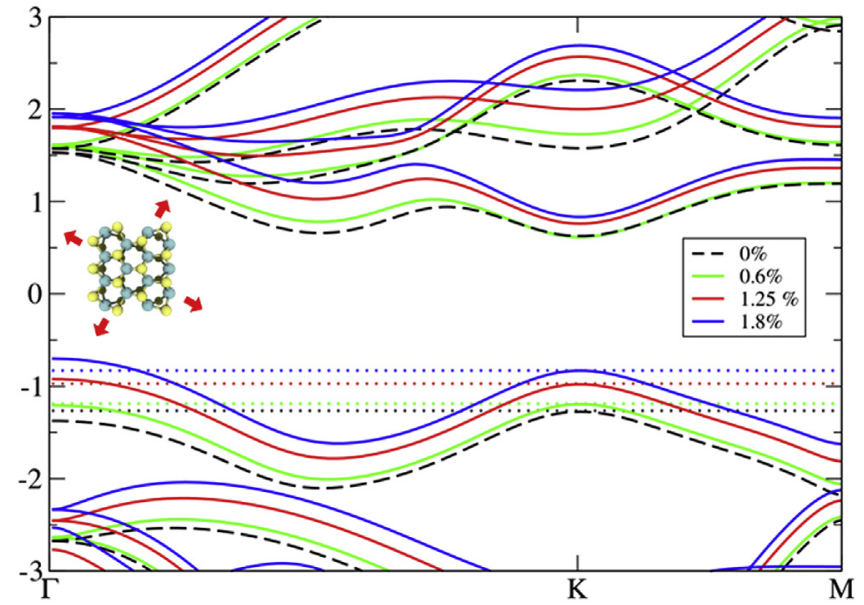

Fig. 5. Calculated band structures of single layered $\mathrm{MoS}_{2}$ under different levels of uniform biaxial tensile strain where the horizontal dotted lines indicate the valence band maximum (in the inset is the atomic model of the strained $\mathrm{MoS}_{2}$ structure where the arrows indicate the direction of the tensile biaxial strain). (A colour version of this figure can be viewed online.)

\section{Materials and methods}

Monolayer graphene was produced following a two-step growth process by using a substrate of $4 \mathrm{H}-\mathrm{SiC}(0001)$. Before starting the graphitization, the substrate was first etched with hydrogen $\left(100 \% \mathrm{H}_{2}\right)$ at $1550{ }^{\circ} \mathrm{C}$ to produce well-ordered atomic terraces of SiC. Subsequently, the SiC sample was heated to $1000^{\circ} \mathrm{C}$ and then further heated to $1550{ }^{\circ} \mathrm{C}$ in an $\mathrm{Ar}$ atmosphere. $\mathrm{MoS}_{2} / \mathrm{SiO}_{2}$ samples were grown by chemical vapor deposition (CVD) in a $1^{\prime \prime}$ quartz tube furnace. Microliter droplets of saturated ammonia heptamolybdate solution were dried onto the corners of a $\mathrm{Si} / \mathrm{SiO}_{2}$ growth substrate that had previously been coated with a layer of sodium cholate ( $1 \%$ solution spin-coated at $4000 \mathrm{rpm}$ for $60 \mathrm{~s}$ ). The growth substrate was placed in the centre of the furnace and heated to $800{ }^{\circ} \mathrm{C}$. A $25 \mathrm{mg}$ sulfur pellet was placed on a piece of silicon and positioned upstream in the furnace such that its temperature was approximately $150^{\circ} \mathrm{C}$. Carrier gas (500 sccm N $\mathrm{N}_{2}$ ) was used to bring sulfur vapor into the furnace for a $30 \mathrm{~min}$ growth period.

XPS experiments were carried out on the TEMPO beamline [50] (SOLEIL French synchrotron facility) at room temperature. The photon source was a HU80 Apple II undulator set to deliver linearly polarized light. The photon energy was selected using a highresolution plane grating monochromator, with a resolving power $\mathrm{E} / \Delta \mathrm{E}$ that can reach 15,000 on the whole energy range (45-1500 eV). During the XPS measurements, the photoelectrons were detected at $0^{\circ}$ from the sample surface normal $\overrightarrow{\boldsymbol{n}}$ and at $46^{\circ}$ from the polarization vector $\overrightarrow{\boldsymbol{E}}$. The spot size was $80 \times 40(\mathrm{H} \times \mathrm{V})$ $\mu \mathrm{m}$. A Shirley background was subtracted in all core level spectra. The C 1s spectra was fitted by a sum of a Gaussian function convoluted with a Doniach-Sunjic lineshape. An asymmetry factor $\alpha$ was used, where $\alpha=0.1 \mathrm{eV}$ (peak G (graphene)) and $\alpha=0 \mathrm{eV}$ (SiC (Silicon Carbide substrate)). The Mo $3 \mathrm{~d}$ and S 2p spectra were fitted by sums of Voigt curves, i.e, the convolution of a Gaussian (of fullwidth at half-maximum GW) by a Lorentzian (of full-width at halfmaximum LW). The LW was fixed at $90 \mathrm{meV}$ for Mo 3d and S 2p [51]. The Mo $3 d$ peak was reconstituted with a $3 d_{5 / 2}: 3 d_{3 / 2}$ ratio of 0.66 and a spin-orbit splitting of $3.10 \mathrm{eV}$. For the $S 2 \mathrm{p}$, a $2 \mathrm{p}_{1 / 2}: 2 \mathrm{p}_{3 / 2}$ ratio of 0.5 and a spin-orbit splitting of $1.19 \mathrm{eV}$ was used. The Raman 
spectra measurements were conducted using a commercial confocal Renischow micro-Raman microscope with a $532 \mathrm{~nm}$ laser in an ambient environment at room temperature. The excitation laser (wavelength $532 \mathrm{~nm}$ ) was focused onto the samples with a spot diameter of $\sim 1 \mu \mathrm{m}$ and incident power of $\sim 3 \mathrm{~mW}$. The integration time was optimized so that the signal-to-noise ratio is acceptable. PL measurements were carried out on the same microscope with a $100 \times$ objective and a Si detector (detection range up to $\sim 2.2 \mathrm{eV}$ ).

First-principles calculations have been performed using a very efficient DFT localized orbital molecular dynamic technique (FIREBALL) [52-55]. Basis sets of $\mathrm{sp}^{3} \mathrm{~d}^{5}$ for $\mathrm{S}$ and Mo were used with cutoff radii (in atomic units) $\mathrm{s}=3.9, \mathrm{p}=4.5, \mathrm{~d}=5.0(\mathrm{~S})$ and $\mathrm{s}=5.0$, $\mathrm{p}=4.5, \mathrm{~d}=4.8(\mathrm{Mo})$. In this study we have considered a standard $(1 \times 1)$ unit cell of $\mathrm{MoS}_{2}$ that has been optimized for different lattice parameters in order to reproduce the strain effect. Finally, a set of 300 special $\mathrm{k}$ points along the $\Gamma-\mathrm{K}-\mathrm{M}$ path has been used for the band structure calculations.

\section{Competing financial interests}

The authors declare no competing financial interests.

\section{Acknowledgements}

The authors would like to thank Dr M. Boutchich and Dr Jaffré Alexandre for the fruitful discussion and the Raman measurements. This work was supported by the H2DH grants. We acknowledge support from Labex "Nanosaclay (Grant No. ANR-10-LABX-0035)". Labex belongs to the public funded Investissements d'Avenir program managed by the French National Research Agency. C.H.N. and A.T.C.J. acknowledge support from the National Science Foundation EFRI-2DARE program, grant number ENG- 1542879.

\section{Appendix A. Supplementary data}

Supplementary data related to this article can be found at http:// dx.doi.org/10.1016/j.carbon.2016.09.041.

\section{References}

[1] G.R. Bhimanapati, et al., Recent advances in two-dimensional materials beyond graphene, ACS Nano 9 (2015) 11509-11539.

[2] Y.-C. Lin, et al., Direct synthesis of van der Waals solids, ACS Nano 8 (2014) 3715-3723.

[3] M. Long, et al., Broadband Photovoltaic Detectors Based on an Atomically Thin Heterostructure, 2016, http://dx.doi.org/10.1021/acs.nanolett.5b04538.

[4] Q.H. Wang, K. Kalantar-Zadeh, A. Kis, J.N. Coleman, M.S. Strano, Electronics and optoelectronics of two-dimensional transition metal dichalcogenides, Nat. Nanotechnol. 7 (2012) 699-712.

[5] K. Roy, et al., Graphene-MoS2 hybrid structures for multifunctional photoresponsive memory devices, Nat. Nanotechnol. 8 (2013) 826-830.

[6] E. Kaxiras, J. Kong, H. Wang, Graphene/MoS2 hybrid technology for large-scale two- dimensional electronics, Nano Lett. 14 (2014) 3055-3063.

[7] A. Ebnonnasir, B. Narayanan, S. Kodambaka, C.V. Ciobanu, Tunable MoS2 bandgap in MoS2-graphene heterostructures, Appl. Phys. Lett. 105 (2014).

[8] W. Jin, et al., Tuning the electronic structure of monolayer graphene/MoS2 van der Waals heterostructures via interlayer twist, Condens. Matter Mesoscale Nanoscale Phys. 201409 (2015) 1-6.

[9] P. Yeh, et al., Direct measurement of the tunable electronic structure of bilayer MoS2 by interlayer twist Direct measurement of the tunable electronic structure of bilayer MoS2 by interlayer twist, Nano Lett. 16 (2) (2016) 953-959, http://dx.doi.org/10.1021/acs.nanolett.5b03883.

[10] G.H. Han, et al., Seeded growth of highly crystalline molybdenum disulphide monolayers at controlled locations, Nat. Commun. 6 (2015) 6128.

[11] C. Riedl, C. Coletti, T. Iwasaki, a. a Zakharov, U. Starke, Quasi-free-standing epitaxial graphene on sic obtained by hydrogen intercalation, Phys. Rev. Lett. 103 (2009) 246804.

[12] E. Pallecchi, et al., High electron mobility in epitaxial graphene on $4 \mathrm{H}^{-}$ $\mathrm{SiC}(0001)$ via post-growth annealing under hydrogen, Sci. Rep. 4 (2014) 4558.

[13] H. Henck, et al., Electrolytic phototransistor based on graphene-MoS2 van der Waals p-n heterojunction with tunable photoresponse $\mathrm{p}-\mathrm{n}$ heterojunction with tunable photoresponse, Appl. Phys. Lett. 109 (2016) 113103.

[14] H. Li, et al., From bulk to monolayer MoS 2: evolution of Raman scattering, Adv. Funct. Mater. 22 (2012) 1385-1390.

[15] D. Pierucci, et al., Band alignment and minigaps in monolayer MoS2-graphene van der Waals heterostructures, Nano Lett. 16 (2016) 4054-4061.

[16] C. Riedl, C. Coletti, U. Starke, Structural and electronic properties of epitaxia graphene on $\mathrm{SiC}(0001)$ : a review of growth, characterization, transfer doping and hydrogen intercalation, J. Phys. D. Appl. Phys. 43 (2010) 374009.

[17] D. Pierucci, et al., Large area molybdenum disulphide-epitaxial graphene vertical Van der Waals heterostructures, Sci. Rep. 6 (2016) 26656.

[18] E. Velez-Fort, et al., Epitaxial graphene on 4H-SiC ( 0001 ) grown under nitrogen flux : evidence of low nitrogen doping and high charge transfer, ACS Nano 6 (2012) 10893-10900.

[19] Kim, I. S. et al. Influence of stoichiometry on the optical and electrical properties of chemical vapor deposition derived MoS2.ACS Nano 1-34

[20] R. Addou, et al., Impurities and electronic property variations of natural MoS 2 crystal surfaces, ACS Nano (2015) 9124-9133.

[21] Y. Guo, D. Liu, J. Robertson, Chalcogen vacancies in monolayer transition meta dichalcogenides and Fermi level pinning at contacts, Appl. Phys. Lett. 106 (2015) 48-53.

[22] a Levasseur, P. Vinatier, D. Gonbeau, X-ray photoelectron spectroscopy: a powerful tool for a better characterization of thin film materials, Bull. Mater Sci. 22 (1999) 607-614.

[23] P.D. Fleischauer, J.R. Lince, A comparison of oxidation and oxygen substitution in MoS 2 solid film lubricants, Tribol. Int. 32 (1999) 627-636.

[24] M.A. Baker, R. Gilmore, C. Lenardi, W. Gissler, XPS investigation of preferentia sputtering of S from MoS 2 and determination of MoS x stoichiometry from Mo and S peak positions, Appl. Surf. Sci. 150 (1999) 255-262.

[25] Z. Yin, et al., Preparation of MoS 2 -MoO 3 hybrid nanomaterials for lightemitting diodes, Angew. Chem. 126 (2014) 12768-12773.

[26] G. Eda, et al., Photoluminescence from chemically exfoliated MoS 2, Nano Lett. 11 (2011) 5111-5116.

[27] H.J. Conley, et al., Bandgap engineering of strained monolayer and bilayer MoS2, Nano Lett. 13 (2013) 3626-3630.

[28] R. Roldan, A. Castellanos-Gomez, E. Capelluti, F. Guinea, Strain engineering in semiconducting two-dimensional crystals, J. Phys. Condens. Matter 27 (2015) 313201.

[29] X. Liu, et al., Rotationally commensurate growth of MoS2 on epitaxial graphene, ACS Nano 10 (2015) 1067-1075.

[30] S. Tongay, et al., Defects activated photoluminescence in two-dimensional semiconductors: interplay between bound, charged, and free excitons, Sci. Rep. 3 (2013) 2657.

[31] H. Nan, et al., Strong photoluminescence enhancement of MoS2 through defect engineering and oxygen bonding, ACS Nano 8 (2014) 5738-5745.

[32] Z. Liu, et al., Strain and structure heterogeneity in MoS2 atomic layers grown by chemical vapour deposition, Nat. Commun. 5 (2014) 5246.

[33] K.F. Mak, et al., Tightly bound trions in monolayer MoS2, Nat. Mater. 12 (2013) 207-211.

[34] D. Sercombe, et al., Optical investigation of the natural electron doping in thin MoS2 films deposited on dielectric substrates, Sci. Rep. 3 (2013) 3489.

[35] N. Scheuschner, et al., Photoluminescence of freestanding single- and fewlayer MoS 2, Phys. Rev. B - Condens. Matter Mater. Phys. 89 (2014) 2-7.

[36] K. Zhang, et al., Self-induced uniaxial strain in MoS2 Mono layers with loca van der Waals-stacked inter layer interactions, ACS Nano 9 (2015) 2704-2710.

[37] Y. Wang, C. Cong, C. Qiu, T. Yu, Raman spectroscopy study of lattice vibration and crystallographic orientation of monolayer mos2 under uniaxial strain, Small 9 (2013) 2857-2861.

[38] W.M. Parkin, et al., Raman shifts in electron-irradiated monolayer MoS 2, ACS Nano 10 (2016) 4134-4142.

[39] M. Sheet, Exceptional Tunability of Band Energy in a Compressively Strained Trilayer, 2013, pp. 7126-7131.

[40] H. Li, et al., Optoelectronic crystal of artificial atoms in strain-textured molybdenum disulphide, Nat. Commun. 6 (2015) 7381.

[41] B. Chakraborty, et al., Symmetry-dependent phonon renormalization in monolayer MoS 2 transistor, Phys. Rev. B - Condens. Matter Mater. Phys. 85 (2012) 2-5

[42] K. Zhou, et al., Raman Modes of MoS 2 used as fingerprint of vander Waals interactions, ACS 8 (2014) 9914-9924.

[43] C. Rice, et al., Raman-scattering measurements and first-principles calculations of strain-induced phonon shifts in monolayer MoS2, Phys. Rev. B. 87 (2013) 081307, http://dx.doi.org/10.110/PhysRevB.87.081307.

[44] Y. Cai, J. Lan, G. Zhang, Y.W. Zhang, Lattice vibrational modes and phonon thermal conductivity of monolayer MoS 2, Phys. Rev. B - Condens. Matter Mater. Phys. 89 (2014) 1-8.

[45] A. Castellanos-gomez, et al., Local strain engineering in atomically thin MoS 2 , Nano Lett. 13 (2013) 5361-5366.

[46] Z. Wang, Q. Chen, J. Wang, Electronic structure of twisted bilayers of graphene/MoS2 and MoS2/MoS2, J. Phys. Chem. C 119 (2015) 4752-4758.

[47] L. Yang, et al., Lattice strain effects on the optical properties of MoS2 nanosheets, Sci. Rep. 4 (2014) 5649.

[48] X. Wang, et al., Ultrasensitive and broadband MoS 2 photodetector driven by ferroelectrics, Adv. Mater. (2015) 6575-6581, http://dx.doi.org/10.1002/ adma.201503340.

[49] W.S. Yun, S.W. Han, S.C. Hong, I.G. Kim, J.D. Lee, Thickness and strain effects on 
electronic structures of transition metal dichalcogenides: 2H-MX2 semiconductors ( $\mathrm{M}=\mathrm{Mo}, \mathrm{W} ; \mathrm{X}=\mathrm{S}, \mathrm{Se}, \mathrm{Te})$, Phys. Rev. B 85 (2012) 033305.

[50] F. Polack, et al., TEMPO: a new insertion device beamline at SOLEIL for time resolved photoelectron spectroscopy experiments on solids and interfaces, AIP Conf. Proc. 1234 (2010) 185-188.

[51] S. Mattila, Ja Leiro, M. Heinonen, T. Laiho, Core level spectroscopy of MoS2, Surf. Sci. 600 (2006) 5168-5175.

[52] J. Lewis, et al., Further developments in the local-orbital density-functionaltheory tight-binding method, Phys. Rev. B 64 (2001) 195103.
[53] J.P. Lewis, et al., Advances and applications in the FIREBALLab initio tightbinding molecular-dynamics formalism, Phys. Status Solidi 248 (2011) 1989-2007.

[54] P. Jelínek, H. Wang, J. Lewis, O. Sankey, J. Ortega, Multicenter approach to the exchange-correlation interactions in ab initio tight-binding methods, Phys. Rev. B 71 (2005) 235101.

[55] O.F. Sankey, D.J. Niklewski, Ab initio multicenter tight-binding model for molecular-dynamics simulations and other applications in covalent systems, Phys. Rev. B 40 (1989) 3979-3995. 